\title{
Efeito do contato dos fungos Beauveria bassiana e Metarhizium anisopliae na ecdise ninfal de Amblyomma cajennense
}

\author{
Effect of contact of the fungi Beauveria bassiana and Metarhizium \\ anisopliae on Engorged Larvae of Amblyomma cajennense
}

\author{
Edson Jesus de Souza, ${ }^{*}$ Rosana Colatino Soares Reis; ${ }^{* \star}$ Vânia Rita Elias Pinheiro Bittencourt ${ }^{\star \star \star}$
}

\begin{abstract}
Resumo
Este experimento teve como objetivo verificar o efeito in vitrode dois isolados de Beauveria bassiana e de três isolados de Metarhizium anisopliae na ecdise larval de Amblyomma cajennense. Foram testadas quatro suspensões com concentrações diferentes de conídios/ml (10E5, 10E6, 10E7e 10E8). Os percentuais médios de muda de larvas ingurgitadas tratadas com as diferentes suspensões foram inferiores ao observado nos grupos controle $(p<0,05)$. Os grupos tratados apresentaram uma percentagem de ecdise inversamente proporcional à concentração de conídios da suspensão $(p<0,05)$. A percentagem mais baixa de ecdise $(<20 \%)$ foi observada no grupo tratado com o isolado M. anisopliae (Ma 959) na concentração de $10 \mathrm{E} 8$ conídios/ml. As CL 50 que interromperam a ecdise variaram de 5,24 x 10E7 até 5,09 x 10E9 conídios/ml entre os diferentes isolados $(p<0,05)$.
\end{abstract}

Palavras-chave: Amblyomma cajennense; Beauveria bassiana; Metarhizium anisopliae; fungos entomopatogênicos; controle microbiano.

\section{Introdução}

Amblyomma cajennense (Fabricius, 1787), amplamente distribuído no continente americano, desde o sul do Texas nos Estados Unidos até a América do Sul, é um parasita que ataca várias espécies animais e o homem (Cooley \& Kohls, 1944; Hoffmann, 1962). Por ser um carrapato que realiza mudas fora do corpo do hospedeiro, necessitando de três hospedeiros para completar seu ciclo, apresenta maior possibilidade de se infectar e transmitir patógenos (Prata et al., 1997), sendo considerado transmissor da Ehrlichiose Bovina (Massard, 1984), Babesiose Eqüina (Horta \& Figueiredo, 1914) e Tifo Exantemático (Monteiro et al., 1931), afora os danos diretos causados pela espoliação sangüínea e lesões cutâneas.

A mortalidade de carrapatos em colônias laboratoriais devido a fungos foi observada por Boycev \& Rizvanov (1960). Mais tarde Lipa (1971) listou 15 fungos encontrados em ovos e larvas de carrapatos. Gorskova (1966), utilizando Beauveria bassiana para promover infecções artificiais em Ixodes ricinus, demonstrou uma diminuição do percentual de eclosão de larvas e uma mortalidade variável de $86 \%$ a $100 \%$ de um grupo de larvas tratadas.
A infecção artificial de ovos, larvas e fêmeas ingurgitadas de Boophilus microplus pelo fungo Metarhizium anisopliae foi descrita por Bittencourt et al. (1994), que observaram elevada mortalidade de larvas, verificando o desenvolvimento deste fungo na hemolinfa desta espécie de carrapato e alterações nas seguintes etapas da fase não-parasitária: período de pré-postura, período de postura, índice de produção de ovos, período de incubação, período e percentual de eclosão.

Barbosa et al. (1997), promovendo a infecção artificial de larvas ingurgitadas de Rhipicephalus sanguineus com o fungo $B$. bassiana, observaram que nas concentrações de 10 E8 conídios/ml de suspensão a mortalidade de larvas ingurgitadas foi de $100 \%$, não ocorrendo, portanto, a muda para ninfa nesse grupo tratado.

Este experimento teve como objetivos verificar o efeito na ecdise in vitro dos fungos $B$. bassiana e $M$. anisopliae, sob forma de suspensão conidial em contato com larvas ingurgitadas de $A$. cajennense e calcular as concentrações letais 50 e 90 para larvas alimentadas desta espécie de carrapato.

\footnotetext{
*Acadêmico de Pós-Graduação em Parasitologia Veterinária - Departamento de Parasitologia Animal (DPA), Instituto de Biologia (IB), UFRRJ. CEP 23851-970, Seropédica, RJ.

** Bolsista de Iniciação Científica / PIBIC-CNPq-DPA /IB / UFRRJ.

*** Professor Adjunto - DPA / IB / UFRRJ.
} 


\section{Material e métodos}

O presente experimento foi desenvolvido na Estação para Pesquisas Parasitológicas W. O. Neitz (EPPWON) do Departamento de Parasitologia Animal da Universidade Federal Rural do Rio de Janeiro (UFRRJ).

Os exemplares utilizados para manutenção da colônia de A. cajennense foram coelhos, Oryctolagus cuniculus (L., 1758), mestiços (Califórnia x Nova Zelândia) com idade entre 60 e 90 dias, de ambos os sexos, com peso inicial entre 1,5 e 2,1 kg, obtidos do setor de Cunicultura do Instituto de Zootecnia da UFRRJ, sem utilização de produtos acaricidas e infestações anteriores com carrapatos. Os animais foram mantidos em gaiolas individuais, onde receberam ração comercial para coelhos (Cargill â) e água ad libitum.

Fêmeas ingurgitadas de $A$. cajennense foram coletadas de cavalos naturalmente infestados e sem contato recente com carrapaticidas. Após a coleta, as mesmas foram lavadas em água corrente, secas, identificadas, pesadas e acondicionadas em placas de Petri por meio de fita adesiva. Após o início da postura os ovos foram pesados, divididos em alíquotas de 200mg e colocados em tubos de ensaio devidamente identificados, em temperatura e umidade relativa controladas $\left(27^{\circ} \mathrm{C}\right.$ e $\left.>80 \% \mathrm{UR}\right)$. Destes ovos originaram as larvas utilizadas neste experimento.

As larvas ingurgitadas usadas no experimento foram obtidas de infestações artificiais em coelhos, seguindo a técnica de Neitz et al. (1971), que se baseia na colocação de um saco feito de tecido fixado ao redor da base das oreIhas dos coelhos, vedando-se o interstício com a pasta Unna aquecida até a fusão. Após a solidificação da pasta, reforça-se a vedação com esparadrapo. Os sacos possuem uma abertura na extremidade distal por onde foram colocadas as larvas para alimentação, sendo a abertura, a seguir, costurada. Após três a cinco dias de ingurgitamento das larvas, estas foram retiradas do saco e divididas em alíquotas de $\mathbf{3 0}$ larvas por tubo de ensaio.

Os isolados de $M$. anisopliae e $B$. bassiana utilizados no experimento foram cedidos pelo Departamento de Entomologia da Escola Superior de Agricultura "Luiz de Queiroz", da Universidade de São Paulo (USP). Foram avaliados três isolados de $M$. anisopliae, denominados $\mathrm{Ma}$ 959 (isolado de carrapato), Ma 319 (isolado de formiga), e Ma E9 (isolado padrão) e dois isolados de B. bassiana, denominados $\mathrm{Bb} 986$ (isolado de carrapato) e $\mathrm{Bb} 747$ (isolado de formiga).

As suspensões de conídios de cada isolado foram preparadas a partir de fungos produzidos em meio de arroz em sacos de polipropileno, utilizando $50 \mathrm{ml}$ de água destilada e três gotas de espalhante adesivo Tween 80â. Cada bioensaio com larvas ingurgitadas foi constituído de um grupo realizado em condições controladas de temperatura e umidade $\left(27 \pm 1^{\circ} \mathrm{C}\right.$ e $>80 \%$ UR), com quatro diferentes tratamentos nas concentrações (10E5, 10E6, 10E7 e
10E8), que foram estabelecidas em pré-experimento e quantificadas em câmara de Neubauer (Bittencourt et al., 1994); e um grupo controle para cada isolado, sendo que para cada tratamento foram feitas 10 repetições e utilizadas 30 larvas ingurgitadas em cada repetição.

Os tubos de ensaio foram vedados na extremidade com algodão hidrófilo e identificados com a concentração e o isolado do fungo utilizado. Após agitação da suspensão a ser testada, cada grupo de larvas recebeu um mililitro da suspensão, sendo mantidas imersas durante cinco minutos. Após esse tempo, o excesso da suspensão foi eliminado através da inversão dos tubos de ensaio, que foram mantidos nas condições de temperatura e umidade já descritas.

A avaliação dos efeitos das diferentes amostras dos fungos $B$. bassiana e $M$. anisopliae sobre larvas ingurgitadas de $A$. cajennense foi realizada através da observação do percentual de ecdise, 15 dias após a exposição aos fungos.

$\%$ Controle $=[($ Número de larvas alimentadas que realizaram muda para ninfa $X 100) / 30]$

Foram feitas análises de variância (ANOVA) para saber se houve variações dentro de um mesmo tratamento e entre os outros tratamentos com os diferentes isolados e concentrações. A ANOVA foi seguida por aplicação do teste de TUKEY $(p<0,05)$ para comparação entre as médias, calculando-se o coeficiente de variação para verificar a precisão dos dados. Para calcular as concentrações letais, CL 50 e CL 90, foi usada a análise de próbites, segundo Litchfield \& Wilcoxon (1949), utilizando o programa "Probit".

\section{Resultados e discussão}

Em relação ao parâmetro percentual de muda de larvas ingurgitadas para ninfas, foram observadas diferenças significativas entre os tratamentos (10E8, 10E7, 10E6, 10E5 e controle) em todos os isolados testados, reduzindo o percentual à medida que se aumentava a concentração de conídios na suspensão (Figura 1).

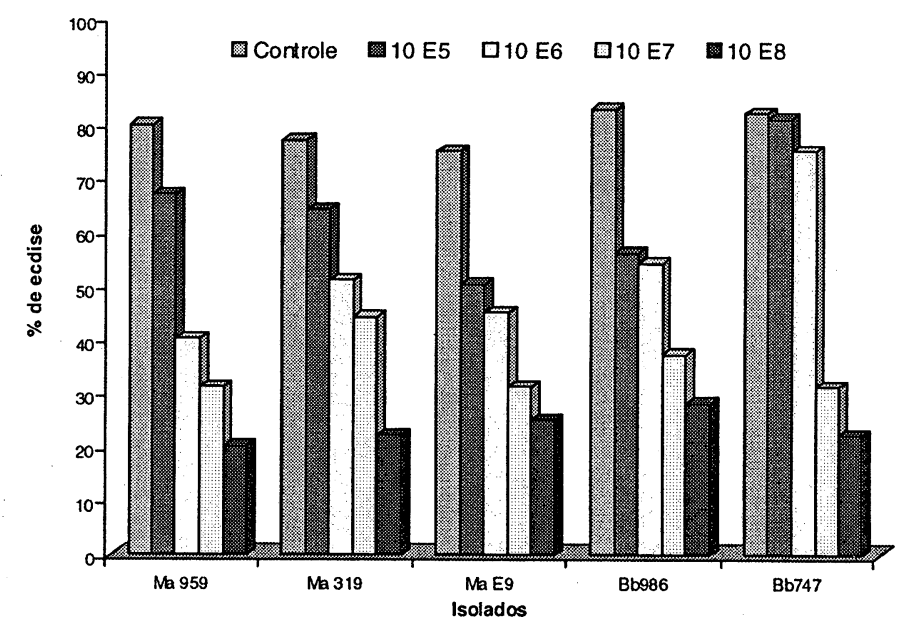

Figura 1: Percentual de ecdise de larvas ingurgitadas de Amblyomma cajennense infectadas com diferentes concentrações dos isolados de fungos entomopatogênicos Metarhizium anisopliae (Ma 959, Ma 319 e Ma E9) e Beauveria bassiana (Bb 986 e Bb 747). 
Os valores de CL 50 e CL 90 encontrados para promover a inibição da ecdise de larvas ingurgitadas, para os diferentes isolados, se encontram na Tabela 1.

Tabela 1: Concentrações letais (CL 50 e CL 90), visando à inibição da ecdise, observadas em larvas alimentadas de Amblyomma cajennense, tratadas com os diferentes isolados de Beauveria bassiana (Bb) e Metarhizium anisopliae (Ma) em condições controladas (Temperatura $27 \pm 1^{\circ} \mathrm{C}$ e Umidade Relativa 80\%)

\begin{tabular}{lcc}
\hline \multirow{2}{*}{ Isolados } & \multicolumn{2}{c}{ Inibição da ecdise } \\
\cline { 2 - 3 } Ma 959 & $\mathrm{CL} \mathrm{50}$ & $\mathrm{CL} \mathrm{90}$ \\
\hline Ma 319 & $5,24 \mathrm{E7}$ & $2,68 \times \mathrm{E} 10$ \\
Ma E9 & $5,09 \mathrm{E9}$ & $3,52 \times \mathrm{E} 15$ \\
$\mathrm{Bb} \mathrm{986}$ & $8,78 \mathrm{E} 8$ & $2,26 \times \mathrm{E} 16$ \\
$\mathrm{Bb} \mathrm{747}$ & $2,94 \mathrm{E} 8$ & $7,64 \times \mathrm{E} 14$ \\
\hline
\end{tabular}

${ }^{1}$ Concentrações em conídios/ml.

Os valores para o percentual de ecdise nos grupos controle variaram de $75 \%$ a $83 \%$ dentre todos os isolados testados; já para os grupos tratados, variaram de $81 \%$ a $20 \%$, conforme as concentrações e os diferentes isolados (Figura 1). Barbosa et al. (1997), avaliando a ação de dois isolados de $B$. bassiana sobre larvas ingurgitadas de Rhipicephalus sanguineus, observaram um percentual de mortalidade das larvas do grupo controle de $24 \%$; já para os grupos tratados, constataram percentuais de 67, 94 e $100 \%$, para os tratamentos $10^{4}, 10^{6} \mathrm{e} 10^{8}$, respectivamente, com o isolado $\mathrm{Bb} 747$. Já para o isolado $\mathrm{Bb} 986, \mathrm{a}$ mortalidade variou de $35 \%$ em $10^{4}$ a $100 \%$ nas concentra- ções de $10^{6} \mathrm{e} 10^{8}$. Os dados apresentados por estes autores são semelhantes aos obtidos no presente trabalho, já que o percentual de mortalidade de larvas ingurgitadas reflete-se inversamente no percentual de ecdise.

Podemos notar que os valores de mortalidade para os grupos tratados, encontrados por Barbosa et al. (1997), refletem percentuais de controle maiores do que os obtidos no presente trabalho, pois em nenhum tratamento observamos um percentual de ecdise de $0 \%$, o que representaria o percentual de mortalidade de $100 \%$, encontrado pelos autores supracitados, nos tratamentos $10^{8}$ conídios $/ \mathrm{ml} \mathrm{em}$ ambos os isolados; essa diferença talvez seja em decorrência de se tratar de espécies diferentes de carrapatos.

São poucos os trabalhos que utilizam controle microbiano pertinentes a esse estágio de desenvolvimento, porém vários autores também verificaram o efeito de fungos entomopatogênicos para larvas não-alimentadas de carrapatos. Bittencourt et al. (1994 e 1996) avaliaram o efeito de $M$ anisopliae e $B$. bassiana, em larvas de Boophilus microplus e verificaram elevada patogenicidade de todos os isolados testados. Monteiro (1997) constatou a elevada mortalidade de larvas não-ingurgitadas de Rhipicephalus sanguineus quando expostas a estes mesmos isolados de entomopatógenos. Boycev \& Rizvanov (1960) verificaram a elevada mortalidade $(93,5 \%)$ de larvas de Ixodes ricinus após a sua imersão numa suspensão de $B$. bassiana. Kaaya et al. (1996), trabalhando com larvas não-ingurgitadas de Rhipicephalus appendiculatus e Amblyomma variegatum, imersas em suspensão com $10^{\circ}$ conídios $/ \mathrm{ml}$ de $B$. bassiana, constataram mortalidade de aproximadamente $100 \%$.

Combase nos resultados aqui apresentados, podemos concluir que os entomopatógenos testados demonstram causar efeito deletério in vitro sobre o estágio de lava ingurgitada de $A$. cajennense, demonstrando, desta forma, seu potencial para utilização em programas de controle desta espécie de carrapato.

\begin{abstract}
This work evaluated the in vitro effects of two isolated of Beauveria bassiana and of three isolated of Metarhizium anisopliae for engorged larvae of Amblyomma cajennense. Four suspensions with different concentrations of conidia/ml $\left(10^{5}, 10^{6}, 10^{7}\right.$ and $\left.10^{8}\right)$ were prepared. Significant differences $(p<0,05)$ were observed among the treatments in the parameter percentage of ecdise in all isolates tested. The percentage of ecdise of engorged larvae treated was smaller than that observed in the control groups, the treated groups presented an ecdise percentage inversely proportional to the conidia concentration of the suspension. The lethal concentrations (LC) 50 found to promote the inhibition of the ecdise of engorged larvae varied of 5,24 $\times 10^{7}$ up to $5,09 \times 10^{9}$ conidia/ml among the different ones isolated.
\end{abstract}

Keywords. Amblyomma cajennense; Beauveria bassiana; Metarhizium anisopliae, entomopathogenic fungi; microbial control.

\section{Referências biliográficas}

BARBOSA, J. V., DAEMON, E., BITTENCOURT, V. R. E. P. \& FACCINI, J. L. H. Efeitos de dois isolados do fungo Beauveria bassiana (Bals.) Vuill. sobre a muda larval e a sobrevivência de ninfas de Rhipicephalus sanguineus (Latreille, 1806) (Acari : Ixodidae). Rev. Bras. Parasitol. Vet., v. 6, n. 1, p. 5356, 1997.
BITTENCOURT, V. R. E. P., MASSARD, C. L. \& LIMA, A. F. 1994. Ação do fungo Metarhizium anisopliae em ovos e larvas do carrapato Boophilus microplus. Rev. Univ. Rural - Série Ciências da Vida, v. 16, n. 1-2, p. 41-47.

BITTENCOURT, V. R. E. P., PERALVA, S. F. S., VIEGAS, E.C., ALVES, S. B. Avaliação dos efeitos do contato de Beauveria bassiana (Bals.) Vuill. com ovos e lavas de Boophilus microplus (Canestrini, 1887) (Acani: Ixodidae). Rev. Bras. Parasitol. Vet., n. 5, p. 81-84, 1996. 
BOYCEV, D. \& RIZVANOV, K. Relation of Botrytis cinerea to ixodids ticks. Zool. Zeitsc. Ukran. n. 39, p. 460, 1960.

COOLEY, R. A \& KOHLS, G. M. The genus Amblyomma (Ixodidae) in the United States. J. Parasitol. n. 30, p. 77-111, 1944.

GORSKOVA, G. J. Reduction of fecundity of ixodid ticks females induced by fungal infection. Vets. Lening Univers., n. 21, p. 13-16, 1966.

HOFFMANN, A. Monographia de los ixodoidea de Mexico, 1 parte. Rev. Soc. Mex. Hist. Nat., n. 23, p. 191-301, 1962.

HORTA, P. P. \& FIGUEIREDO, A . S. Nutaliose dos equideos em Minas Gerais (A mijadeira dos poldrinhos). Rev. Vet. e Zoot., n. 4, p. 3, 1914.

KAAYA, G. P., MWANGI, E. N. \& OUNA, E. A. Prospects for Biological Control of Livestock Ticks, Rhipicephalus appendiculatus and Amblyomma variegatum, Using the Entomogenous Fungi Beauveria bassiana and Metarhizium anisopliae. J. of Invert. Pathol., n. 67, p. 1520, 1996.

LIPA, J. J. Microbial control of mites and ticks. In: BURGES, H. D. \& HUSSEY, N. W. ed. Microbial control of insects and mites. 2. ed. London, Academic Press, p. 357-374, 1971.
LITCHFIELD, J. T. Jr. \& Wilcoxon, F. Simple method of fitting dose-effect curve. J. of Pharmac. and Exper. Therap., n. 95 , p. 99-113, 1949.

MASSARD, C. A. Ehrlichia bovis (Donatien \& Lestoquard, 1936) diagnóstico, cultivo in vitro e aspectos epidemiológicos em bovinos no Brasil. Tese (Doutorado) - Universidade Federal Rural do Rio de Janeiro. 113 p.

MONTEIRO, J. L., FONSECA, F. \& PRADO, A. Pesquisas epidemiológicas sobre o tifo exantemático de São Paulo. Mem Instit Butantã, n. 6, p. 139-173, 1931.

MONTEIRO, S. G. Ação dos fungos Metarhizium anisopliae (Metschnikoff, 1879) Sorokin, 1883 e Beauveria bassiana (Balsamo) Vuillemin, 1912, sobre o carrapato Rhipicephalus sanguineus (LATREILLE, 1806) em diferentes temperaturas. Tese (Mestrado) - Universidade Federal Rural do Rio de Janeiro, $43 p$.

NEITZ, W. O; BOUGHTON, F. \& WALTERS, H. S. Laboratorial investigation on the life cicle of the karoo paralysis ( Ixodes rubicundus Neumann, 1904). Onderstepoort. J. Res. n. 38 , p. 215-224, 1971.

PRATA, M. C. A.; ALONSO, L. S. \& SANAVRIA, A. Parâmetros biológicos do estágio larval de Amblyomma cajennense (Fabricius, 1787) (Acari : Ixodidae) em coelhos. Rev. Bras. Ciênc. Vet., n. 1, p. 5-8, 1997.

\section{H HEXÁGONO}

Química e Equipamentos para Laboratórios

\section{Distribuidor Merck}

Rua Souza Barros, $\mathrm{n}^{2} 560$ - Engenho Novo 20961-150 - Rio de Janeiro, RJ
Tel.: (21) 501-4536

Fax: (21) 501-3723 